EASD

Procedia
EURODYN 2020

XI International Conference on Structural Dynamics M. Papadrakakis, M. Fragiadakis, C. Papadimitriou (eds.) Athens, Greece, 23-26 November 2020

\title{
A NUMERICAL INVESTIGATION OF NEW ALGORITHMS FOR THE DRIVE-BY METHOD IN RAILWAY BRIDGE MONITORING
}

\author{
L. Bernardini ${ }^{1}$, M. Carnevale $^{2}$, C. Somaschini ${ }^{1}$, K. Matsuoka ${ }^{3}$, A. Collina ${ }^{1}$ \\ ${ }^{1}$ Politecnico di Milano \\ Dipartimento di Ingegneria Meccanica \\ via La Masa 1, 20156 Milano, Italy \\ e-mail: andrea.collina@polimi.it \\ ${ }^{2}$ Università degli Studi di Pavia \\ Dipartimento di Ingegneria Industriale e dell'Informazione \\ via Ferrata 5, 27100, Pavia, Italy \\ e-mail: marco.carnevale@unipv.it \\ ${ }^{3}$ Railway Technical Research Institute, \\ Railway Dynamics Division, \\ 2-8-38, Hikari-cho, Kokubunji-shi Tokyo 185-8540 Japan
}

Keywords: Railway bridges; Drive-by monitoring; Truss structures; Steel structure; Indirect methods for SHM; Continuous wavelet transform (CWT).

\begin{abstract}
Railway Infrastructure Manager increasingly demand for new and effective method for the monitoring of infrastructure health, so that a relevant effort is being placed in the development of condition based monitoring and maintenance techniques for railway infrastructure. This includes track geometry, overhead contact line and the structural health of railway bridges. This paper proposes an algorithm for the bridge monitoring of railway bridges, based on accelerations measured on board train (drive-by method). The diagnostic algorithm is developed and validated based on the results of a $3 D$ numerical model simulating the dynamic interaction of the bridge/track/train structure. The analysis is focused on steel truss bridges, on which the degradation of the connection of one diagonal element is simulated (e.g. lose bolts, deteriorated welding). In comparison with previous research works, this paper tries to overcome the need of comparison to a reference signal corresponding to the healthy bridge, which is the technique on which most of literature papers rely on. This is achieved by exploiting the roll component of the train (difference between right and left acceleration), excited as a consequence of the presence of the defect which generates an asymmetry in the bridge behavior. The numerical results show good accuracy in damage identification and location, good robustness to vehicle speed, to the presence of electrical noise and, in the case of a relevant damage, to track irregularity.
\end{abstract}




\section{INTRODUCTION}

Condition based monitoring and maintenance techniques for railway infrastructure are hot research topics for academies and railway industries, regarding track geometry, overhead contact line, and the structural health of railway bridges [1],[2],[3]. Most of railway bridges were designed several decades ago, in the middle of the last century or even before, when loading conditions and traffic volumes were much different and lighter than today [4],[5]. Some of these bridges may also have undergone deterioration and corrosion during time, and they could now be structurally deficient.

The direct monitoring of a single bridge with dedicated instrumentation [6], which is a common practice in the case of main or high-speed lines, is not a feasible prospective for the entire regional and inter-regional railway lines of a country, due the huge number of bridges and the high costs related. Current bridge maintenance relying on visual inspections can be effective if clear protocols are followed, according to the typology of bridge. The obvious drawback of visual inspection is the dependence on to the experience of the inspector and also on the position of the damage, which often happens to be on a hidden member. Moreover, the time interval between inspections may also depend on the availability of personnel. For these reasons, in the last years, indirect methods have been looked for, relying on measurements acquired on the vehicle which crosses the structure. They are commonly named as drive-by methods [1]. The fundamental concept behind this approach is that a structural damage causes a change in the physical and mechanical properties of the bridge, and the bridge can be directly excited by the train passage. As a consequence, a change in the dynamic behavior of the vehicle interacting with the structure can be observed too. The advantage is that, fitting simple measurement set-up on a normal operating train, it is possible to acquire data in an almost continuous way, so that a proper trend of bridge condition could be in principle obtained for building a data base.

Drive-by methods have been mainly developed for road applications (highway bridges), but in fewer cases to railways too. They can be divided in the following two main categories [1]: modal parameters and non-modal parameters. The former, consisting in the identification of natural frequencies [7], [8], [9], [10], damping estimation methods [11] and identification of bridge mode shapes [12], [13], are demonstrated to be effective up to limited speeds (maximum $60 \mathrm{~km} / \mathrm{h}$ ) and, in the first two cases, they are not able to identify the exact location of a defects. These techniques are not trivial to be put in practise, and most of them were only demonstrated through numerical simulations. Moreover, their outcome can be affected by environmental conditions like temperature, especially in the case of damping and natural frequencies [14],[15],[16].

Non modal damage detection methods do not explicitly require the computation of bridge dynamic properties, but focuses on the bridge deflection under passing loads (e.g. apparent profile [17], [18] or change of curvature[19],[20]) or on the dynamic response of the vehicle crossing the bridge [21],[22],[3]. This paper focuses on the latter method, which is thought to be more promising and easy-to-implement, even if at the time being it is mostly investigated by numerical analysis. Most of the cases presented in the literature rely on the comparison of accelerations signals, gathered from the train crossing the bridge, to a reference acceleration signal corresponding to the healthy case. Accelerations are low pass filtered to cancel the vehicle and bridge natural frequencies and to isolate the quasi-static component of the signals. The current open points and drawbacks of these methods are related to the small magnitudes of the signals involved, and the need to know the precise position of the train on the bridge to perform the difference against the reference signal [23],[24]. This position may be achieved through balises or transpoder able to identify and transmit on-board train the position of the 
bridge. On the other hand, this method seems to be effective even for vehicle speeds close to the common commercial speeds of commuter trains, and not too sensitive to track irregularities [3].

Another possibility to exploit the dynamic response of the vehicle crossing the bridge consists in the comparison of signals measured in the same train's run on different bogies (i.e. leading or trailing), or on different side of the vehicle if the type of defect mainly induces variation on one side of the bridge.

Following the above mentioned research line, and in particular the use of Continuous Wavelet Transform [25], this paper investigates the possibility to overcome the need of comparison to a reference signal corresponding to the healthy bridge. This result is achieved by exploiting the roll component of the bogie (difference between right and left accelerations), excited as a consequence of the presence of a defect which generates an asymmetry in the bridge behavior. The results try to put a step forward with respect to previous research work, all relying on the comparison with a baseline of the healthy bridge [2],[3]. The analysis is performed through numerical simulations carried out with a $3 \mathrm{D}$ numerical model for the train/track/bridge interaction [26] of a single span bridge and on a multi-span bridge.

The paper is organized as follows: Section 2 describes the 3D model for the simulation of the bridge/track/train interaction, and the simulation plan. Section 3 describes the proposed algorithm and the results achieved for a single span bridge. Section 4 summarizes the results in the case of a multi-span bridge. Finally, conclusions are drawn in Section 5.

\section{NUMERICAL MODEL AND PLAN OF SIMULATIONS}

The dynamic simulations of the bridge/track/train interaction are carried out with the software developed at the Department of Mechanical Engineering of Politecnico di Milano [26]. The bridge models considered are steel Warren open truss bridges with medium-span, nonballasted track, with timber sleepers directly fastened to the girder. This configuration is selected as a first example, since it has more probability to show differences related to the loss of symmetry in the structure. As a first step of the work, a single line bridge is considered, with the track in the middle of the bridge structure. Two cases are analyzed: a single spanbridge (properties in Figure 1), and a multi-span bridge composed of 4 spans with the same properties of the single span model. This second case is investigated to evaluate the possibility of detecting defects on a damaged span by comparison with the records taken on the other spans.

\begin{tabular}{ll}
\hline Span length & $21.42[\mathrm{~m}]$ \\
height & $3.71[\mathrm{~m}]$ \\
width & $4.5[\mathrm{~m}]$ \\
$1^{\text {st }}$ bending mode & $16 \mathrm{~Hz}$ \\
$1^{\text {st }}$ torsional mode & $19.5 \mathrm{~Hz}$ \\
\hline \multicolumn{2}{l}{ Span dimensions and natural frequencies }
\end{tabular}

\begin{tabular}{llllll}
\hline \multirow{2}{*}{ Element } & $\begin{array}{c}\text { Section } \\
\text { area }\end{array}$ & $\begin{array}{c}\text { Polar } \\
\text { moment }\end{array}$ & $\begin{array}{c}\text { Torsion } \\
\text { constant }\end{array}$ & $\begin{array}{c}\text { Principal } \\
\text { moment }\end{array}$ & $\begin{array}{c}\text { Principal } \\
\text { moment }\end{array}$ \\
\cline { 2 - 6 } & $\left.\mathrm{A} \mathrm{m}^{2}\right]$ & $\mathrm{I}_{\mathrm{p}}\left[\mathrm{m}^{4}\right]$ & $\mathrm{I}_{\mathrm{t}}\left[\mathrm{m}^{4}\right]$ & $\mathrm{I}_{2}\left[\mathrm{~m}^{4}\right]$ & $\mathrm{I}_{3}\left[\mathrm{~m}^{4}\right]$ \\
\hline 1 & 0.0143 & $3.910^{-4}$ & $1.5410^{-6}$ & $2.1210^{-4}$ & $1.810^{-4}$ \\
2 & 0.0148 & $3.510^{-4}$ & $1.9110^{-6}$ & $2.1410^{-4}$ & $1.410^{-4}$ \\
3 & 0.00615 & $2.010^{-4}$ & $9.210^{-7}$ & $1.6510^{-4}$ & $4.110^{-5}$ \\
4 & 0.0013 & $1.0310^{-4}$ & $4.610^{-7}$ & $8.210^{-5}$ & $2.110^{-5}$ \\
5 & 0.0167 & $9.110^{-4}$ & $6.610^{-7}$ & $8.9710^{-4}$ & $1.910^{-5}$ \\
6 & 0.0048 & $2.510^{-4}$ & $6.410^{-7}$ & $2.310^{-4}$ & $2.210^{-5}$ \\
\hline \multicolumn{6}{c}{ Section properties. Area and moments of Inertia }
\end{tabular}

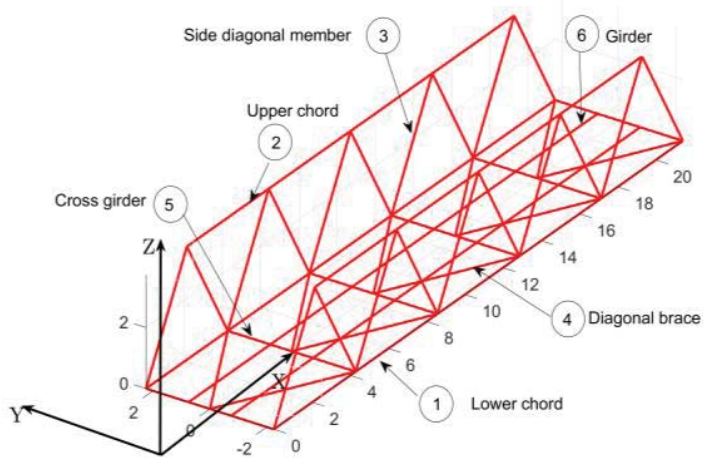

Figure 1: Single span bridge. 
Two ballasted modules of length $29.4 \mathrm{~m}$ are added before and after the bridge (track properties as in reference [3]), to represent more realistic transients when the train enters and exits the bridge. The damage introduced in the bridge model represents a full or partial degradation of the fastenings in correspondence of the connection between the diagonal member (labelled with 3 in Figure 1) and the lower chord (labelled with 1 in Figure 1). The full damage is modeled by removing the diagonal element from the finite element model and applying a concentrated mass at the connection of the damaged element with the upper chord, to maintain its weight. The partial damage, which represents a more realistic damage condition in practice, is modeled through a reduction of the Young modulus of the damaged diagonal member. This is equivalent to considering a spring at the location of the fastening, in series with the involved diagonal element. Three different defect conditions have been studied, with a reduction of the Young Modulus equal to $30 \%, 50 \%$ and $70 \%$ in the damaged diagonal member. Different damage positions along the span are considered, to study whether and how damage location affects the accuracy of its detection.

Simulations of two different commuter trains have been considered, namely CSA and TSR model. They are respectively built with bogies shared between adjacent carbodies (CSA), and with the standard two-bogies configuration for each carbody, double-deck type (TSR). Their properties are described in reference [3], and not repeated here for sake of conciseness. Simulations for each train type are carried out with the train crossing the bridge at four different constant velocities, in the range of $80 \mathrm{~km} / \mathrm{h}-140 \mathrm{~km} / \mathrm{h}$. These speeds are near to the maximum commercial speeds of these kind of trains (respectively 140 for TSR and $160 \mathrm{~km} / \mathrm{h}$ for CSA), so that if satisfactory results are achieved, the desirable result of no traffic delays would result in the line.

The dynamic response of the train is measured at the bogie frame level, on the right and left side, in correspondence of the wheelset. The position of the accelerometer is of primary importance: location on axle box increases the sensitivity to defect, but involves a higher level of acceleration. This higher level of acceleration, requiring a larger full-scale value of the transducer, implies a lower sensitivity [3]. On the other hand, the location of the sensors on the bogie frame takes advantage of the effect of mechanical filtering from the primary suspension, attenuating high frequency components in the acceleration and enabling the use of more sensitive accelerometers. The drawback consists in the fact that sensors on the bogie frame are more distant from the location of the defect, so that the vibration path associated to the defect might be detected with more difficulties. Location on the bogie frame is investigated in the present paper, also considering the advantages in terms of maintenance operation of the train and the measurement set-up itself.

Since the proposed method for damage identification is based on the difference between the right and left hand side accelerations, a different irregularity profile is adopted on left and right rails, so as to simulate a more realistic case. Both vertical and roll track irregularities are considered, the total track profile being a composition of the two contributions. Profiles are obtained starting from the power spectral density functions provided by the standard ORE B176, and obtaining different level of irregularities (the highest level is reported in Figure 2). The level of irregularity is considered to be higher in the ballasted modules before and after the bridge, and it is attenuated along the bridge extension, where a non-ballasted track is present. This is a reasonable assumption considering that on the bridge the only permanent displacement is due to the dimension variation of the timber sleepers. 


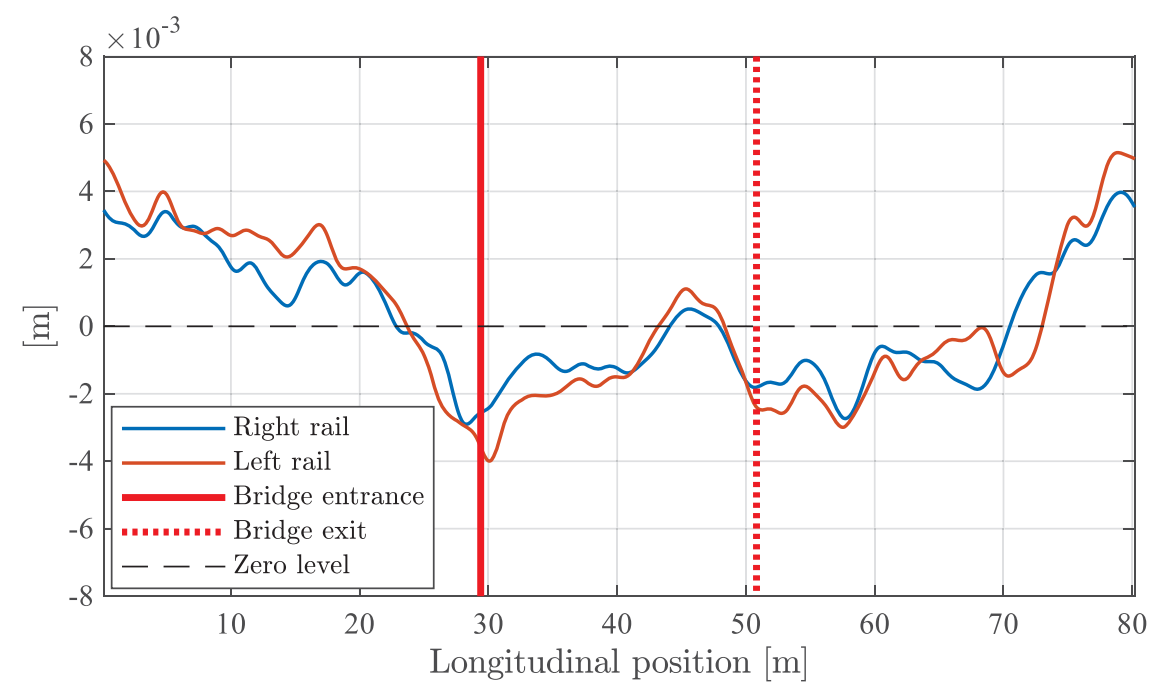

Figure 2: Vertical irregularities on left and right rails.

All the above mentioned parameters are changed individually during the simulations, varying only one parameter at once to investigate its effect on the outcomes of the proposed algorithm.

\section{RESULTS FOR THE SINGLE SPAN BRIDGE}

The presence of a defect on the bridge side generates an asymmetry in its behavior under the loads of the passing train. Figure 3 shows the deflection of the healthy (Figure 3a) and damaged (Figure $3 \mathrm{~b}$ ) bridges under the train load, with the black arrows highlighting the positions of the two axles of the leading bogie of the CSA train at a specific frame. In Figure $3 b$ the damage is placed on the sixth diagonal member of the right side wall, right in the middle of the two arrows: due to the damage presence, the bridge deflection loses the symmetry characterizing the healthy structure. This asymmetry can be exploited to detect the presence of a defect, by analyzing the difference between the right and left hand side accelerations of the leading bogie.

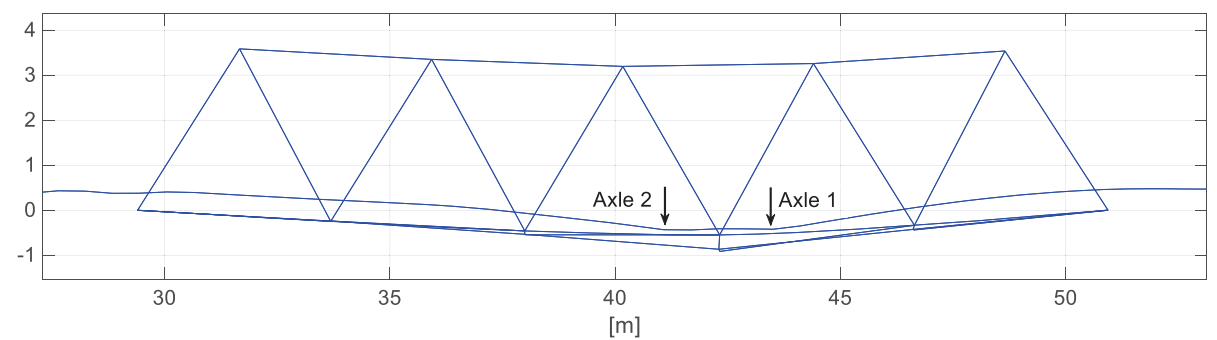

(a)

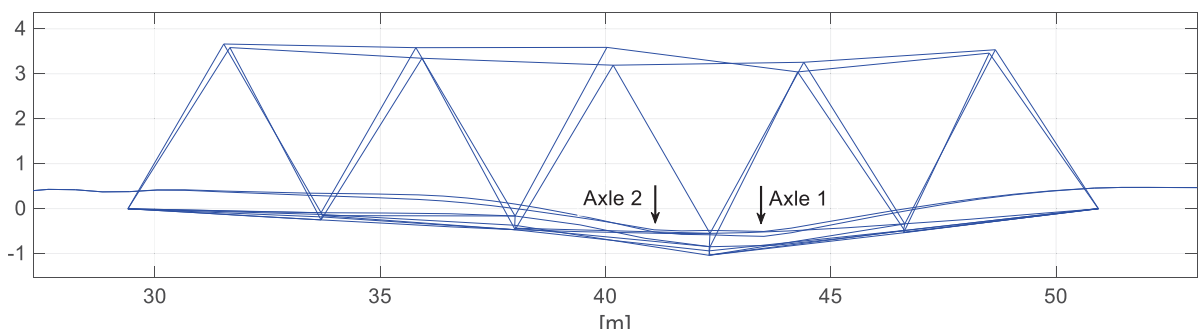

(b)

Figure 3: Bridge deflection under the train load (a) Healthy bridge. (b) Damaged bridge. 
The processing algorithm consists in the application of the Continuous Wavelet Transform (CWT) to the difference of accelerations measured on the front side of the leading bogie, in correspondence of the right and left axle-boxes. The Huang-Hilbert transform was tested too on the same signal, obtaining consistent results between the two methods. Accelerations on the leading bogie are among the most suitable for diagnostic purposes, for the reason reported in [3]: the leading bogie interacts with an unperturbed bridge, so that differences in accelerations generated by a defect can be identified more precisely; bogie accelerations benefit from the filtering action provided by the primary suspension, which removes disturbances in the higher frequency range, mainly generated by track irregularities.

The possibility to detect a damage on the bridge based on the difference of right and left acceleration can be potentially affected by longitudinal and roll irregularity of the track, which may hide the presence of a damage. In order to assess the robustness of the proposed method in regard to this aspect, a different irregularity profile is adopted for left and right rails, as already described in Figure 2.

Figure 4 reports, as an example, the difference of the right and left side accelerations on the leading bogie of the CSA train at $100 \mathrm{~km} / \mathrm{h}$, corresponding to the case of total damage. A random disturbance is added to represent an electric noise, obtained from a Gaussian distribution with standard deviation equal to $10 \%$ of the RMS of the original acceleration signal. In the figure, the outer lines represent the start (continuous line) and end (dotted line) of the bridge, whereas the dashed vertical lines in the middle represent the location of the defect. The maximum magnitude of the raw signal is out of the damaged zone, and cannot be of course used as a diagnostic indicator. Wavelet coefficients are therefore computed on this signal, adopting a real-valued Morlet Wavelet.

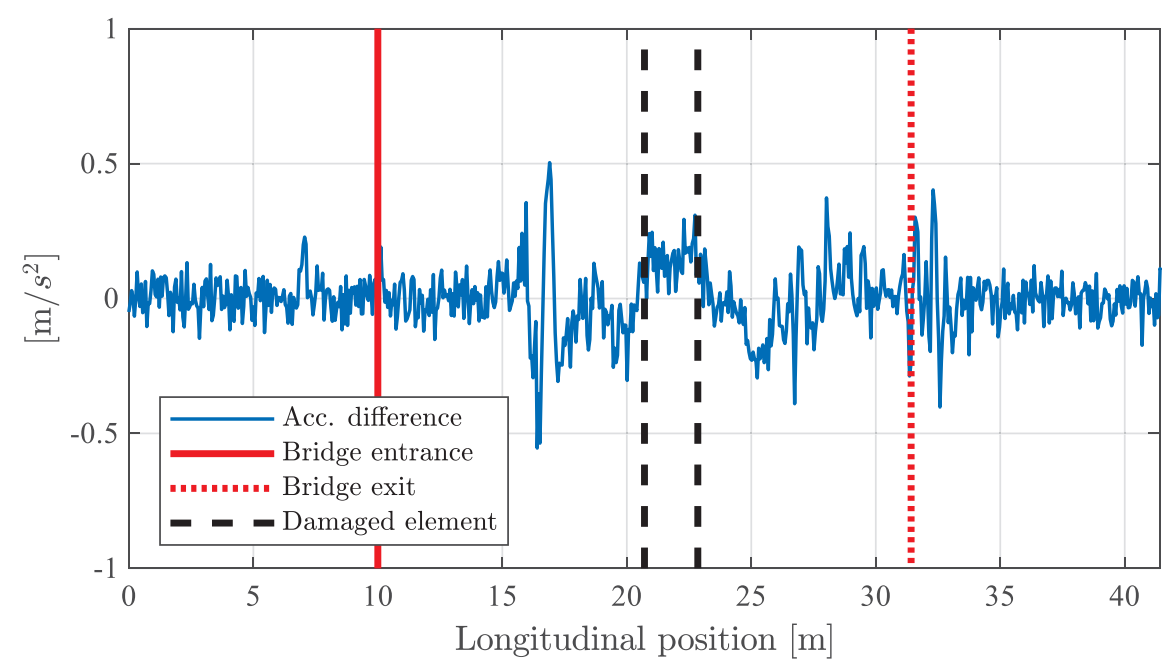

Figure 4: Difference between right and left accelerations on the leading bogie. Train speed $100 \mathrm{~km} / \mathrm{h}$.

Figure 5 reports the comparison of the module (absolute value) of the CWT coefficients in the case of the damaged bridge (Figure 5a) and of the healthy bridge (Figure 5b). The presence of the defect can be identified around the pseudo-frequency of $3.85 \mathrm{~Hz}$, which corresponds to the frequency of the first peak obtained by applying a Fast Fourier Transform on the signal of Figure 4. The presence of the defect can be identified in a relatively low frequency range, where the CWT coefficients show peak values which are not present in the case of the healthy bridge. The reported results correspond to the highest level of irregularity adopted, which is the reason why several peaks in the CWT coefficients are visible at higher frequencies. With a reduction of $-70 \%$ of the Young modulus of the diagonal element the damage is still clearly 
identified, whereas for lower entity of the damage (e.g. reduction of 50\% of the Young modulus), the presence of irregularity tends to hide the presence of a defect. The exploitability of this method is therefore a compromise between the effect induced by the defect, depending on the defect entity, and the presence of track irregularity.

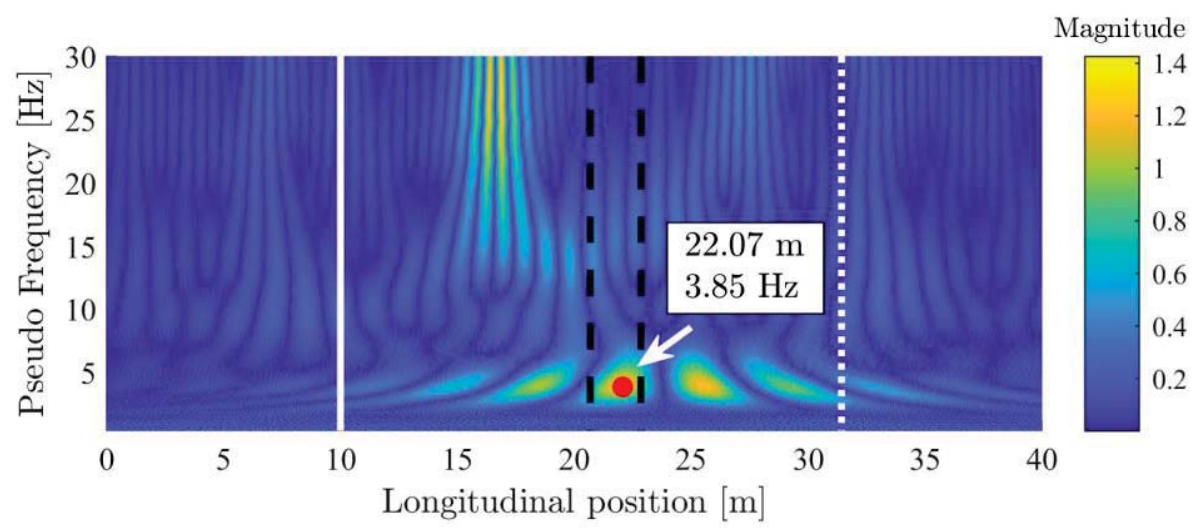

(a)

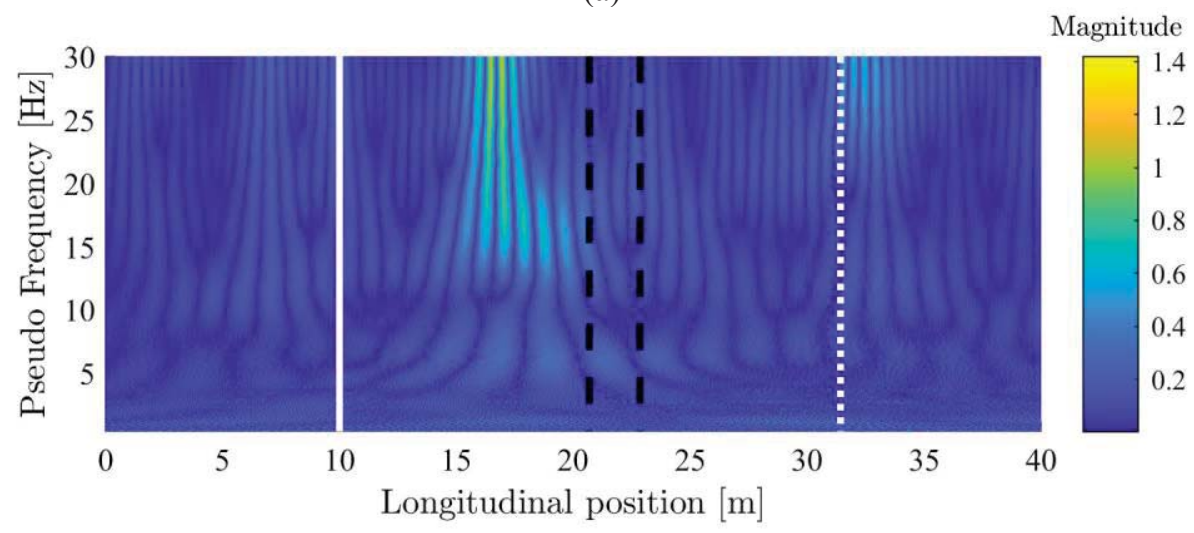

(b)

Figure 5: Module of the CWT coefficients, difference between the right and left side bogie accelerations (a) Damaged bridge, total damage. (b) Healthy bridge.

\section{SIMULATION RESULTS OF MULTI-SPAN BRIDGE}

The previous paragraph described the possibility to detect the presence of a relevant defect by exploiting the asymmetry in the bridge/train behavior. As a further step the case of a multispan bridge is used to investigate the possibility of identifying a defect through the comparison of indices computed with reference to different spans. Figure 6 represents the scheme of the FEM model developed. The damaged span is highlighted by the black dashed box, whereas the remaining spans are assumed to be healthy. The damage element on span 3 is around the middle span (i.e. from $10.71 \mathrm{~m}$ to $12.85 \mathrm{~m}$ on a span length of $21.42 \mathrm{~m}$ ).

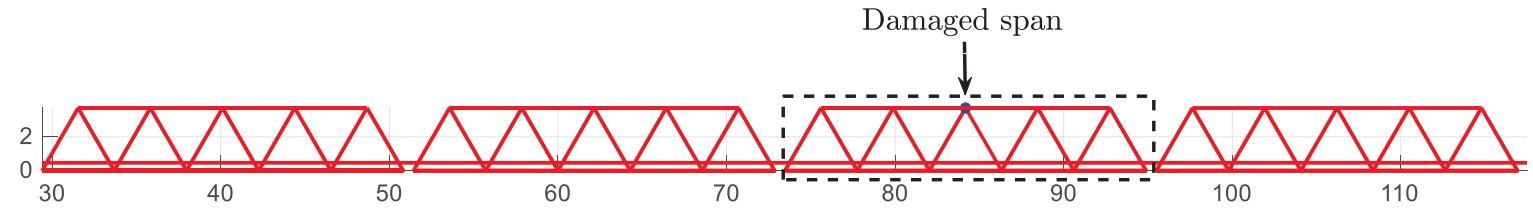

Figure 6: Lateral view of the multi-span bridge simulated. 
The irregularity profile adopted is different for each span and for the left and right rails. Figure 7 represents the total irregularity profile on left and right rail, both outside and inside of the bridge area.

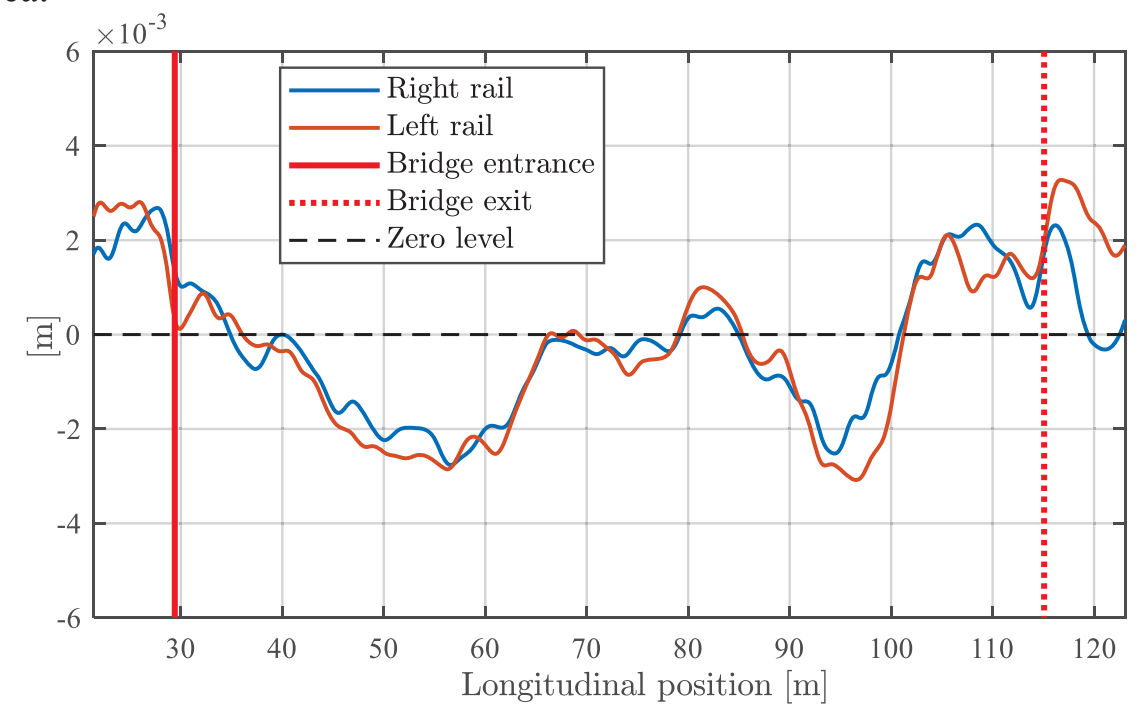

Figure 7: Total irregularity profile for left and right rail.

The acceleration data corresponding to each span can be analyzed separately, assuming to be able to separate the time histories of the four spans by the adoption of a precise GPS signal (e.g. EGNOSS). The difference between right and left bogie accelerations corresponding to each span are reported in Figure 8 for the simulation case with train speed set to $100 \mathrm{~km} / \mathrm{h}$ and a total damage on the bridge. In the case of the damaged $3^{\text {rd }}$ span it is possible to identify an oscillating shape, which is related to the difference between right and left side accelerations generated by the presence of the defect. The peculiarity of this signal shape can be caught by CWT.

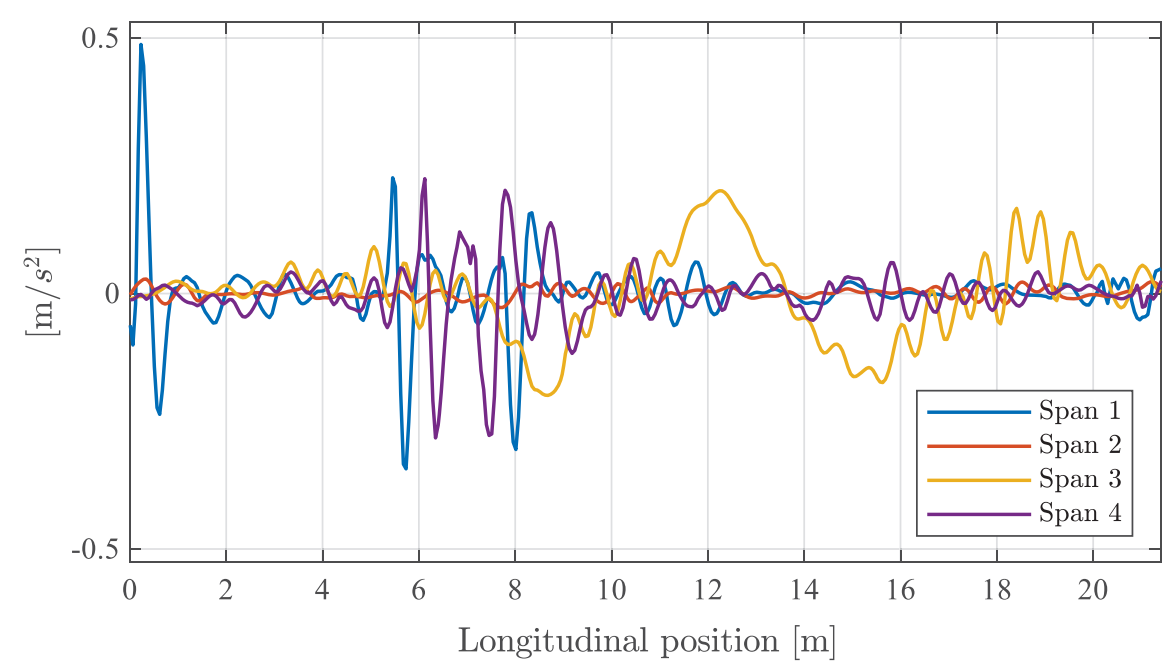

Figure 8: Front bogie accelerations difference, span from 1 to 4.

Figure 9 shows the CWT coefficients obtained with a Morlet wavelet for each single span. Once again it is possible to observe that the defect on the third span is properly caught, also by comparison with the analysis of the other spans. The defect can be properly identified for the total damage, or partial damage with a relevant reduction of the Young modulus in the diagonal element, whereas in the case of small damages it is hidden by track irregularity. 

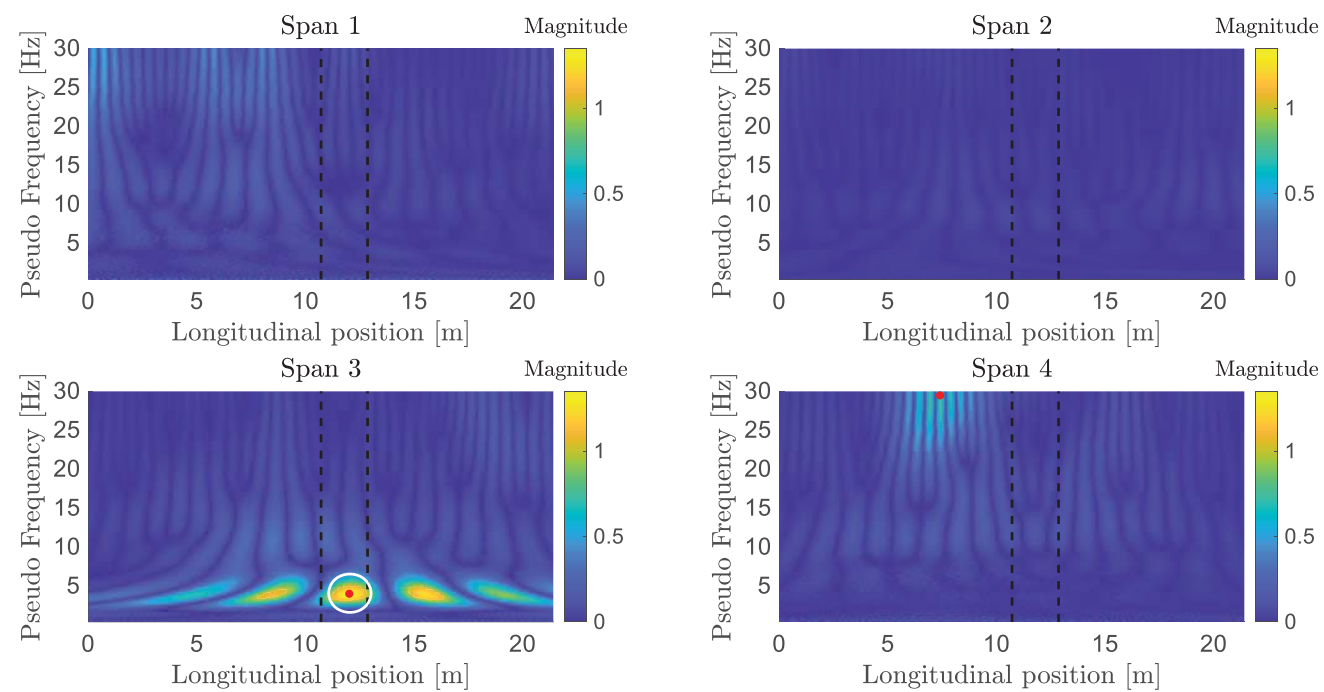

Figure 9: Module of the CWT coefficients evaluated on the difference of right and left hand side bogie accelerations (leading bogie, in correspondence of the front axle-boxes).

\section{CONCLUSIONS}

The paper focuses on a drive-by method for the diagnostics of railway bridges in commuter lines. It puts forward the idea that the presence of a defect on the bridge may introduce an asymmetry in the structure, which may consequently cause an asymmetry in the dynamic behavior of the train. This asymmetry can be caught by analyzing the difference between the right and left side accelerations measured on the frame of the first bogie of the train, and without the need of comparison with a baseline signal referring to the bridge in healthy condition.

The analysis is carried out through a full 3D model for the simulation of the dynamic behavior of the bridge/track/train system, in the presence of both vertical and roll track irregularity to represent a scenario close to reality. A different profile of the left and right rails may hide indeed the presence of a defect. The defect is inserted by considering the failure, or a percentage ineffectiveness, of the connection of one diagonal member of the side frame in a Warren, open type, medium-span bridge.

The difference between right and left side accelerations corresponding to the leading bogie of the train at the leading axle are analyzed through Continuous Wavelet Transform. The results show that when the simulated defect is relevant (i.e. detachment of one diagonal member in the truss bridge, or strong reduction of its Young modulus) the defect can be properly identified even with a high level of irregularity, but, for lower entities of the damage, the difference of irregularity between right and left track can hide the presence of the defect. The exploitability of this method is therefore dependent on the entity of the defect and on the severity of track irregularity.

\section{REFERENCES}

[1] Abdollah Malekjafarian, Patrick J. McGetrick and Eugene J. Obrien (2014), A review of indirect bridge monitoring using passing vehicles (2014), Hindawi Publishing Corporation Shock and Vibration Volume 2015, Article ID 286139. 
[2] Amerio, L., Carnevale, M., Collina, A. Damage detection in railway bridges by means of train on-board sensors: A perspective option (2018) The Dynamics of Vehicles on Roads and Tracks, 2, pp. 1215-1220.

[3] Carnevale, M., Collina, A., Peirlinck, T. A feasibility study of the drive-by method for damage detection in railway bridges (2019) Applied Sciences (Switzerland), 9 (1), art. no. 160, DOI: 10.3390/app9010160.

[4] S. L. Davis and D. Goldberg, The Fix We're In For: The state of our Nation's bridges 2013, Transportation for America, Washington, DC, USA, 2013.

[5] A. Znidari c,V.Pakrashi, E.O'Brien, andA.O'Connor, "A review of road structure data in six European countries," Proceedings of the ICE: Urban Design and Planning, vol. 164, no. 4,pp. 225-232, 2011.

[6] Matsuoka, K., Kaito, K., Sogabe, M. Bayesian time-frequency analysis of the vehiclebridge dynamic interaction effect on simple-supported resonant railway bridges (2020) Mechanical Systems and Signal Processing, 135, art. no. 106373. DOI: 10.1016/j.ymssp.2019.106373

[7] Y.B. Yang, C. W. Lin, and J. D. Yau, "Extracting bridge frequencies from the dynamic response of a passing vehicle," Journal of Sound and Vibration, vol. 272, no. 3-5, pp. 471-493, 2004.

[8] Dionysius M. Siringoringo and Yozo Fujino (2010), Estimating bridge fundamental frequency from vibration response of instrumented passing vehicle: Analytical and Experimental Study, Advances in Structural Engineering Vol. 15 No. 32012

[9] Y. B. Yang, Y. C. Li, K. C. Chang, Using two connected vehicles to measure the frequencies of bridges with rough surface: a theoretical study, Acta Mech 223, 1851-1861 (2012) DOI 10.1007/s00707-012-0671-7

[10] Chul-Woo Kim, Kai-Chun Chang, Patrick John McGetrick, Shinichi Inoue, and Souichiro Hasegawa (2017), Utilizing moving vehicles as sensors for bridge condition Screening-A Laboratory Verification, Sensors and Materials, Vol. 29, No. 2 (2017) $153-163$

[11] R. O. Curadelli, J. D. Riera, D. Ambrosini, and M. G. Amani, "Damage detection by means of structural damping identification," Engineering Structures, vol. 30, no. 12, pp. 3497-3504, 2008

[12] Y. Zhang, L. Q. Wang, and Z. H. Xiang, "Damage detection by mode shape squares extracted from a passing vehicle," Journal of Sound and Vibration, vol. 331, no. 2, pp. 291-307, 2012.

[13] Eugene J. OBrien and Abdollah Malekjafarian A mode shape-based damage detection approach using laser measurement from a vehicle crossing a simply supported bridge, Struct. Control Health Monit. 2016; 23:1273-1286

[14] Eugene J. OBrien, Abdollah Malekjafarian, Arturo González, Application of Empirical Mode Decomposition to drive-by bridge damage detection (2017), European Journal of Mechanics - A/Solids · January 2017

[15] Fan, W., Qiao, P.Z., (2011). Vibration-based damage identification methods: a review and comparative study. Struct Health Monit 10, 83-111. 
[16] Qiao, P.Z., Cao, M.S., 2008. Waveform fractal dimension for mode shape-based damage identification of beam-type structures. Int J Solids Struct 45, 5946-5961.

[17] Paraic Quirke, Cathal Bowe, Eugene J. OBrien, Daniel Cantero, Pablo Antolin, Jose Maria Goicolea, Railway bridge damage detection using vehicle-based inertial measurements and apparent profile, Engineering Structures 153 (2017) 421-442

[18] Ahmed Elhattab, Nasim Uddin, Eugene Obrien (2016), Drive-by bridge damage monitoring using Bridge Displacement Profile Difference, J Civil Struct Health Monit DOI 10.1007/s13349-016-0203-6

[19] Eugene J. OBrien, Daniel Martinez, Abdollah Malekjafarian, Enrique Sevillano Damage detection using curvatures obtained from vehicle measurements, Journal of Civil Structural Health Monitoring · July 2017

[20] Abdollah Malekjafarian, Daniel Martinez, and Eugene J. Obrien (2018), The feasibility of using laser doppler vibrometer measurements from a passing vehicle for bridge damage detection, Hindawi Shock and Vibration Volume 2018, Article ID 9385171, 10 pages

[21] David Hester \& Arturo González (2015) A bridge-monitoring tool based on bridge and vehicle accelerations, Structure and Infrastructure Engineering, 11:5, 619-637, DOI:10.1080/15732479.2014.890631

[22] González, A.; Hester, D. An investigation into the acceleration response of a damaged beam-type structure to a moving force. J. Sound Vib. 2013

[23] Cathal Bowe, Paraic Quirke, Daniel Cantero, and Eugene J. Obrien, Drive-by structural health monitoring of railway bridges using train-mounted accelerometers, Conference Paper · May 2015 DOI: 10.7712/120115.3490.751

[24] David Hester, Arturo González (2017) A discussion on the merits and limitations of using drive by monitoring to detect localised damage in a bridge, Mechanical Systems and Signal Processing 90 (2017) 234-253

[25] Paul C. Fitzgerald, Abdollah Malekjafariana, Daniel Cantero, Eugene J. OBrien, Luke J. Prendergastc, Drive-by scour monitoring of railway bridges using a wavelet-based approach, Engineering Structures 191 (2019) 1-11

[26] Bruni, S.; Collina, A.; Corradi, R.; Diana, G. Numerical simulation of train-trackstructure interaction for high speed railway systems, IABSE Symposium, Antwerp 2003, Belgium. 